$$
\text { CONF- } 780157-1
$$

To be presented at the Workshop on

LBL-8252

Physics and Engineering in Computerized

Tomography, ỤC, Irvine, January 17-19. 1978

3-D OBJECT RECONSTRUCTION EMISSION AND TRANSMISSION TOMOGRAPHY WITH I.IMITED ANGULAR INPUT

K. C. Tam, V. Perez-Mendez and B. Macdonald

October 1978

Prepared for the U. S. Department of Energy under Contract W-7405-ENG-48

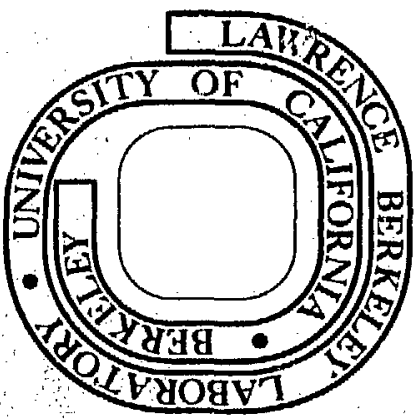




\title{
3-D OBJECT RECONSTRUCTION IN EMISSION AND TRANSMISSION TOMOGRAPHY WITH LIMITED ANGULAR INPUT
}

\author{
K. C. Tam, V. Perez-Mendez and B. Macdonald \\ Lawrence Berkeley Laboratory and University of California, \\ San Francisco
}

\section{ABSTRACT}

The effects of the angular range of data taking in reconstructions in planar positron cameras using the deconvolution method and the matrix method, respectively, are investigated. It is found that in the absence of any a priori information there are undetermined components in the reconstruction if the field of view of the positron camera is limited. However, most of the undetermined components are recovered in the case in which the transverse spacing of the object is discrete, and all of them are recovered if the fact that the object extent is finite is utilized. It is concluded that the two reconstruction methods are mathematically equivalent. The results obtained can be applied to other transmission and emission imaging devices. 


\title{
3-D OBJECT RECONSTRUCTION EMISSION AND TRANSMISSION
}

\section{TOMOGRAPHY WITH LIMITED ANGULAR INPUT}

\author{
K. C. Tam, V. Perez-Mendez and B. Macdonald \\ - Lawrence Berkeley Laboratory and University of California,
}

San Francisco

\section{SUMMARY}

Most emission and transmission imaging methods involve taking data in a continuous range of angles or series of discrete angles. In this paper we investigate the relationship between the angular range of data taking and the quality of the reconstructions by studying the typical problem of imaging in planar positron cameras (Fig. 1). Different algorithms for reconstructing the the object distribution $\rho(\underline{r})$ from the data solve the integral equation

$$
\phi(\underline{r})=\int \rho\left(\underline{r}^{\prime}\right) \phi_{0}\left(\underline{r}-\underline{r}^{\prime}\right) d^{3} \underline{r}^{\prime}
$$

in different ways ( $\phi$ is the scalar field constructed from the data, $\phi_{0}$ is the point response function). One such algorithm Fourier transforms equation (1) to the frequency space (k-space) and soives for $R(\underline{k})$, the Fourier components of $\rho(\underline{r})$. Another algorithm Fourier transforms equation (1) in the $x$ and $y$ dimensions only and solves the resulting integral equation in the $z$ dimension for every spatial frequency $\left(k_{x}, k_{y}\right)$. We shall refer to these two methods as the deconvolution method [1,2] and the matrix method [3] respectively.

In the case where the range of integration in equation (1) covers all space, there would be a region in frequency space where $\Phi_{0}(\underline{k})$ (the fourier transform of $\left.\phi_{0}(\underline{r})\right)$ is zero if $\phi_{0}$ does not cover the full angular range, as shown in Fig. 2. The consequences are that the corresponding components of $R(\underline{k})$ cannot be determined, and that the integral equation in the $z$ coordinates 
in the matrix method does not admit unique solutions. We demonstrated the effect of the size of the camera angle by reconstructing the isotope distribution from the data generated by the same phantom in a two-sided, a four-sided and a six-sided camera whose detectors correspond to one, two and three pairs of opposite faces of a cube with the object at its center. Table I shows the optimum value of $x^{2}$, a measure of the deviation of the reconstruction from the phantom, for each configuration.

The undetermined fourier components can be recovered partly or completely if a priori constraints are utilized. If we assume that the distribution of $\rho(\underline{r})$ in the z-dimension is discrete, most of the zero components of $\Phi_{0}(\underline{k})$ will become non-zero through sampling. Thus, the corresponding components of $R(\underline{k})$ will be recovered in the deconvolution method, and the degree of undeterminacy will be correspondingly reduced in the integral eqliation in the $z$ coordinate of the matrix method.

If we make use of the fact that the object is finite in extent, all the undeterminacy is removed. Thus, in the deconvolution method, the facts that (1) the Fourier transform of a finite object is an entire function, and (2) an entire function can be continued throughout the whole complex plane from a knowledge of the function on any finite continuous line segment, make it possible to recover the undetermined components of $R(\underline{k})$. In the case of the matrix method, it can be shown that the integral operator in the $z$ coordinate becomes positive-definite when the range of integration is finite, with the consequence that unique solutions exist for each $k_{x} \neq{ }^{\prime} 0$; the undetermined components at $k_{x}=0$ can be filled in by analytic continuation from $k_{x} \neq 0$. In our work, an iterative scheme was employed to extend $R(\underline{k})$ obtained from the deconvolution method beyond the deconvolution region. The value of $x^{2}$ is significantly improved (Table II). 
Table I

Deconvolution Without Iterations

\begin{tabular}{|l|c|c|c|}
\hline Number of sides & 2 & 4 & 6 \\
\hline Optimum $x^{2}$ & 1.000 & 0.442 & 0.435 \\
\hline
\end{tabular}

Table II

Deconvolution With Iterations

\begin{tabular}{|c|c|c|c|c|}
\hline $\begin{array}{l}\text { Number of } \\
\text { Iterations }\end{array}$ & 0 & 5 & 10 & 15 \\
\hline $\mathrm{x}^{2}$ & 1.000 & 0.431 & 0.389 & 0.381 \\
\hline
\end{tabular}


$\gamma$ DETECTOR

$\gamma$ DETECTOR

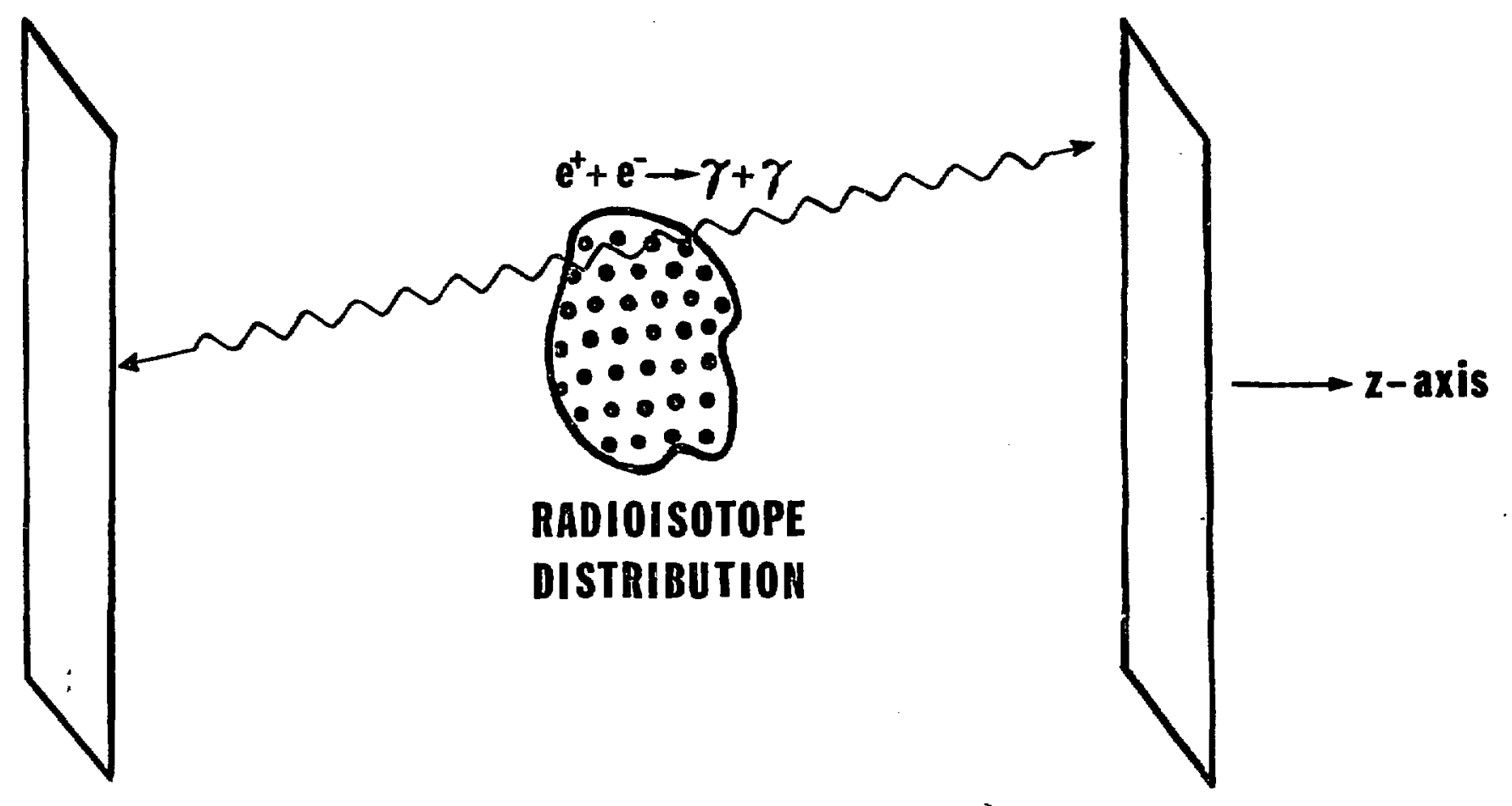

in

XBL-7810-11996

Figure 1. Schematic figure of planar positron camera. 
